\title{
PARAFUSOS ILIOSACRAIS SÃO REALMENTE SEGUROS?
}

\author{
ARE ILIOSACRAL SCREWS REALLY SAFE?
}

\begin{abstract}
Marcus Vinicius Dias', Flavio Goldsztajn², Flavio Ribeiro³, João Matheus Guimarães ${ }^{4}$, José Afraneo Grizendi ${ }^{3}$ Leonardo Rosa Rocha ${ }^{5}$, Marcos Correia ${ }^{6}$, Tito Henrique Rocha
\end{abstract}

\section{RESUMO}

Objetivo: Avaliar a segurança do uso de fixação iliosacral com parafusos em pacientes com lesão do anel pélvico. Métodos: Trata-se de um trabalho restrospectivo analisando por tomografia computadorizada a topografia de 60 parafusos inseridos em 46 pacientes portadores de lesões do anel pélvico e operados no INTO-RJ, no período de 2006 e 2010. Resultados: Em 21,7\% dos casos o parafuso encontrava-se fora do corredor de segurança descrito em literatura. Destes, 77\% estavam associados a uma redução insatisfatória do anel pélvico. Não houve casos de lesão neurovascular em nossa série. Conclusão: $\mathrm{O}$ uso de parafusos ílio-sacrais é uma técnica segura, pois mesmo em casos onde o parafuso não respeitou uma topografia ideal, não houve complicações significativas associadas a este método. A má redução do anel pélvico é um fator muito importante associado ao mau posicionamento dos parafusos.

\section{ABSTRACT}

Objective: To evaluate the safety of iliosacral screw fixation in pelvic injuries. Methods: A retrospective study based on computer tomography evaluation of screw topography and its relationship with the so called pelvic safe corridor. Results: We evaluated the topography of 60 screws inserted in 46 patients sustaining pelvic injuries which were operated in the INTO from 2006 to 2010. In 21.7\% of cases the screw was located outside the safe corridor. $77 \%$ of these misplaced screws were associated with an unsatisfactory reduction of the pelvic injury. Conclusions: In our study, a significant rate of misplaced screws in relationship with the safe corridor has been pointed out and in most of these cases an insufficient reduction of the pelvis was also identified. We conclude that iliosacral fixation is a safe adjuvant method for pelvic fixation since in our series even with a relative high incidence of misplacements, no neurovascular injuries have been recorded in association with this technique.

\section{INTRODUÇÃO}

A fixação iliosacral com parafuso é um método bem estabelecido para a estabilização para as lesões posteriores do anel pélvico ${ }^{(1)}$.

Inserido de forma percutânea após uma redução fechada ou aberta, o parafuso iliosacral pode ser fixado ao corpo de S1, de S2 ou transfixando o ilíaco contralateral, passando por esses dois corpos vertebrais ${ }^{(2)}$.
Dado o carater percutâneo da técnica, esse método depende de imagem fluoroscópica de bom padrão de qualidade, no intuito de se posicionar o parafuso dentro do "corredor de segurança anatômico", evitando-se, dessa forma, potenciais lesões iatrogênicas às estruturas neurovasculares adjacentes à fixação sacral ${ }^{(3)}$.

Falta de planejamento pré-operatório; utilização inadequada da técnica; imagens fluoroscópicas de baixa

1 - Médico Assistente do Centro de Pelve e Acetábulo do Instituto Nacional de Traumatologia e Ortopedia - INTO, Rio de Janeiro, Brasil.

2 - Chefe do Centro de Pelve e Acetábulo do Instituto Nacional de Traumatologia e Ortopedia - INTO, Rio de Janeiro, Brasil.

3 - Médico Assistente do Centro de Trauma do Instituto Nacional de Traumatologia e Ortopedia - INTO, Rio de Janeiro, Brasil.

4 - Chefe do Serviço do Instituto Nacional de Traumatologia e Ortopedia - INTO, Rio de Janeiro, Brasil.

5 - Chefe do Centro de Trauma do Idoso do Instituto Nacional de Traumatologia e Ortopedia - INTO, Rio de Janeiro, Brasil.

6 - Diretor do Hospital de Traumato Ortopedia Dona Lindu, Paraíba do Sul, Brasil.

7 - Chefe da Coordenação de Desenvolvimento Institucional do Instituto Nacional de Traumatologia e Ortopedia - INTO, Rio de Janeiro, Brasil.

Trabalho realizado no Centro de Pelve e Acetábulo do Instituto Nacional de Traumatologia e Ortopedia do Ministério da Saúde (INTO-MS), RJ, Brasil.

Correspondência: Marcus Vinícius Fernandes Dias - Rua Presidente Backer, 25/902, Icaraí-Niterói-RJ; CEP: 24220-045. E-mail: mvortopedia@hotmail.com

Trabalho recebido para publicação: 15/03/11, aceito para publicação: 15/04/11. 
qualidade per operatória; particularidades do paciente como obesidade e dismorfismos sacrais; e falta de redução anatômica da lesão, são alguns dos fatores que influenciam negativamente o resultado do método, dificultando a inserção do parafuso iliosacral em seu corredor de segurança, e a tornando um grande desafio para os cirurgiões de pelve que lidam com essas lesões ${ }^{(2,4)}$.

\section{OBJETIVOS}

O presente trabalho visa a estudar a posição dos parafusos iliosacrais em relação ao seu corredor de segurança na pelve, por meio de avaliação tomografica pós-operatória, avaliando a influência da qualidade da redução da lesão posterior do anel pélvico na posição destes parafusos no corredor de segurança.

\section{MATERIAL E METODOS}

Foram avaliadas, retrospectivamente, todas as fraturas do anel pélvico com lesão posterior que foram tratadas cirurgicamente no Instituto Nacional de Ortopedia e Traumatologia (INTO) pela técnica de parafuso iliosacral percutâneo, no período de Fevereiro de 2006 a Janeiro de 2010. Todos os pacientes foram avaliados inicialmente por uma análise radiográfica pelas incidências de Pennal e Sutherland ${ }^{(5)}$ (antero-posterior, inlet e outlet) e por meio de tomografia computadorizada helicoidal (TC), com reconstrução em corte axial, sagital e coronal de $1,3 \mathrm{~mm}$.

\section{Critérios de Inclusão}

Todos os pacientes, independentemente do sexo; independentemente da idade, desde que esqueleticamente maduros; independentemente do padrão de fratura; e que possuíam lesão do anel pélvico, submetidos ao tratamento cirúrgico pela técnica de parafuso iliosacral percutâneo, com redução aberta ou fechada da lesão, no INTO, no intervalo de tempo analisado pelo nosso trabalho.

\section{Critérios de Exclusão}

Pacientes não operados ou pacientes em que foram utilizadas outras técnicas de fixação da lesão posterior; pacientes em que não foi realizada a TC de controle pós operatória; pacientes com perda de seguimento ambulatorial.

A presença ou não de fixação da lesão anterior e o seu tipo não foram levados em consideração.
A técnica utilizada de inserção de parafusos, nos pacientes em decúbito dorsal, levou em consideração os parâmetros anatômicos descritos por Letournel e popularizados por Matta ${ }^{(6)}$, em 4 incidências fluoroscópicas, a saber: perfil verdadeiro do sacro, ântero-posterior da pelve, além do inlet e outlet view. Em todos os pacientes, foram utilizados parafusos canulados $8.0 \mathrm{~mm}$ da Stryker ${ }^{\circledR}$. Em nenhum de nossos casos foi utilizada monitoração per operatória eletroneurofisiológica.

O passo a passo da técnica já foi anteriormente publicado em nosso meio por esse mesmo grupo, e sua descrição, pormenorizada, foge ao escopo do presente trabalho $^{(7)}$.

Todos os pacientes foram submetidos a controle de imagem pós-operatório recente (até o terceiro dia após a cirurgia) de TC helicoidal, com reconstrução nos planos axial, coronal e sagital.

A tomoografia computadorizada pós operatória foi analisada por 2 cirurgiões familiarizados com fratura do anel pélvica, os quais levaram em conta a posição do parafuso em relação ao corredor de segurança e a qualidade da redução da lesão. No caso em que houve discordância entre os avaliadores quanto à posição dos parafusos e/ou em relação a qualidade da redução, um terceiro avaliador, igualmente familiarizado com esse tipo de lesão, foi escolhido para ser consultado e o seu julgamento foi o considerado para fim de análise dos resultados.

Consideramos como adequados os parafusos que em todo o seu trajeto permaneceram totalmente dentro do osso, desde a asa do ilíaco, asa do sacro, corpo de S1 ou de S2 e não tocaram ou violaram, a cortical superior do sacro ou o espaço discal L5/S1; não tocaram ou violaram a cortical anterior do corpo de S1 e/ou S2; não violaram ou tocaram os limites dos forames neurais e/ou não violaram ou tocaram os limites do canal raquiano $^{(4)}$.

Consideramos como redução satisfatória todos os casos em que o desvio residual, mensurado pela TC, da lesão,em qualquer um dos planos, foi menor ou igual a $10 \mathrm{~mm}^{(8)}$.

Consideramos como redução insatisfatória, os casos em que o desvio residual foi superior a $10 \mathrm{~mm}^{(8)}$.

Dos 47 pacientes operados no período, apenas um foi excluído por não ter sido encontrada a tomografia computadorizada pós operatória para fim de análise. Dos 46 pacientes de nossa casuística, avaliamos o posicionamento de 60 parafusos. 
Os resultados foram avaliados por média e desviopadrão. O presente trabalho se encontra em conformidade com a declaração de Helsinki da Associação Médica Mundial

\section{RESULTADOS}

Foram 46 pacientes, sendo $38(82,6 \%)$ do sexo masculino, 8 (17,4\%) do feminino; a idade mínima foi de 17 anos e a máxima 69 anos (média de 31 anos +- 2,6 anos); e a distribuição quanto ao padrão de lesão, segundo a classificação de Tile $^{(9)}$, foi:

C1.2: 30 casos $(65,3 \%)$

C1.3: 10 casos $(21,7 \%)$

C2: 3 casos $(6,5 \%)$

C3: 3 caso $(6,5 \%)$

Dos 30 pacientes com lesão tipo C1.2, em 28 (93,3\%) casos, os parafusos foram inseridos em S1 e em 2 (6,7\%) casos apenas em S2.

Em 4 dos 10 pacientes com lesão do tipo C1-3, foram inseridos parafusos em $\mathrm{S} 1$ e $\mathrm{S} 2$, consecutivamente, sendo que nos outros 6 casos apenas em S1.

Nos 3 pacientes com lesão do tipo C2 foi inserido 1 parafuso no corpo de S1 em cada lado da lesão.

Dos 3 casos em que a lesão foi classificada como do tipo C3, foi inserido 1 parafuso no corpo de S2 em cada lado da lesão, em 1 dos casos; sendo que nos 2 casos restantes foram inseridos 2 parafusos no corpo de S1 (1 em cada lado) e 2 parafusos no corpo de S2 (1 de cada lado) .

\section{Dos 60 parafusos analisados}

- Em relação à posição no corredor de segurança: 47 (78,3\%) adequados e 13 (21,7\%) inadequados;

- Em relação à qualidade da redução da lesão: $44(73,3 \%)$ satisfatórias e $16(26,7 \%)$ insatisfatórias;

- Correlação entre os parafusos em posição inadequada, em relação ao corredor de segurança, com a qualidade de redução:

Em $10(77 \%)$ dos 13 casos em que o parafuso estava em uma posição considerada inadequada, a qualidade da redução foi considerada insatisfatória.

\section{DISCUSSÃO}

Pacientes com fraturas e/ou luxações pélvicas são um desafio para o traumatologista. Entre as razões para tal dificuldade, podem ser citadas o desvio, a cominuição, a lesão de partes moles, as lesões neurovasculares, a imagem fluoroscópica inadequada, entre outras. ${ }^{(2,3,8)}$

A fixação com parafuso iliosacral percutâneo tem se tornado um método popular para o tratamento das lesões posteriores do anel pélvico. Tal método é rápido, com mínima perda sanguínea, e requer pequenas incisões. Esses fatores podem diminuir problemas como: sangramentos com repercussão clínica, infecções e complicações de partes moles ${ }^{(1,10)}$.

Entretanto, uma inserção segura do parafuso iliosacral depende de uma anatomia óssea pélvica favorável e uma correta compreensão, por parte do cirurgião, desta anatomia e sua correlação com as imagens obtidas pela fluoroscopia $^{(6,12)}$.

A inserção incorreta destes parafusos coloca em risco estruturas nobres neurovasculares que se encontram adjacentes ao corredor de segurança em que o procedimento é realizado ${ }^{(11)}$.

Entre os fatores que contribuem para um posicionamento inadequado do parafuso e "estreitam" o corredor de segurança, destacam-se: os dismorfismos da parte superior do sacro e a má qualidade da redução da lesão ${ }^{(5,8,9)}$.

Idealmente, o parafuso deve ser posicionado no centro dos corpos vertebrais de S1 e S2, devendo ser evitada a penetração do recesso da asa do sacro, dos forames das raízes nervosas, bem como do canal raquiano, do espaço discal L5/S1 e da cortical anterior do sacro. Os parâmetros para isso foram determinados por Carlson et $a l^{(4)}$.

Em trabalho em laboratório utilizando cadáveres, Barrick et $a l^{(10)}$ utilizaram parafusos iliosacrais guiados por TC. Encontraram 37,5\% de parafusos fora do corredor em S2 e nenhum parafuso fora do corredor em S1.

Em nossa casuística, 21,7\% dos parafusos estavam em posição considerada inadequada. Ao contrário de Barrick et $a l^{(10)}$, não tivemos nenhuma violação do corredor dos parafusos colocados em S2, e sim em S1.

Templeman et $a l^{(12)}$, chamaram a atenção para a avaliação pós operatória por TC destes pacientes, e para a proximidade dos parafusos com estruturas nobres adjacentes.

Em sua série de 57 parafusos, em 5 casos de parafusos inseridos em S1 houve penetração ou violação da cortical do forame de S1; em 1 caso o parafuso saiu pela cortical anterior da asa do sacro e entrou novamente no corpo sacral (fenômeno chamado de in-out-in por Chip Routt $\left.^{(11)}\right)$; em 1 caso o parafuso violou a cortical anterior do sacro; e em 1 caso houve a violação da cortical anterior do canal raquiano. 
Em nossa série, 6 parafusos violaram os limites do forame de S1; em 5 houve violação da cortical anterior do corpo de $\mathrm{S} 1$ e em 2 casos ocorreu o fenômeno do in-out-in.

Em 1 caso de Templeman et $a l^{(12)}$, houve lesão iatrogênica que acometeu a raíz de $\mathrm{S} 1$. Curiosamente, no caso em questão, o parafuso inserido encontrava-se totalmente dentro da zona de segurança, tendo sido atribuída, como causa da lesão, a manipulação da fratura que se situava na zona II de Dennis do sacro.

Em nossa casuística, não houve lesão pos-operatória neurovascular. Tal achado revela que, a despeito de uma incidência relativamente alta de parafusos fora do corredor de segurança, tal técnica pode ser considerada segura.

Templeman et $a l^{(12)}$, não levam em consideração a qualidade da redução das lesões em seu trabalho.

Para tal fato, Reilly et al ${ }^{(8)}$, chamam a atenção. Em trabalho em laboratório, esses autores discutem a influência da qualidade de redução das fraturas sacrais no corredor de segurança dos parafusos iliosacrais. Segundo os seus achados, a área de secção transversa do corredor diminui $30 \%, 56 \%, 81 \%$ e $90 \%$, para desvios residuais de $5,10,15$, e $20 \mathrm{~mm}$, respectivamente. Ainda, segundo esses autores, em $50 \%$ dos espécimes com $15 \mathrm{~mm}$ de desvio e em $66 \%$ dos com $20 \mathrm{~mm}$, não foi possível a colocação de 2 parafusos, simultâneos, totalmente dentro dos limites ósseos. Em 17\% dos espécimes com desvios de $15 \mathrm{~mm}$ e em $50 \%$ dos com $20 \mathrm{~mm}$, a área de secção transversa foi insuficiente para conter um simples parafuso iliosacral.

Esses achados corroboram nossos resultados. Dos 13 parafusos que de alguma forma violavam o corredor de segurança, em $10(77 \%)$ a redução da lesão foi considerada insatisfatória.

Para Reilly et $a l^{(8)}$, a redução anatômica da fratura é obrigatória para a utilização da técnica de parafusos iliosacrais percutâneos. E nos casos em que o desvio residual é superior a $10 \mathrm{~mm}$, as estruturas neurovasculares adjacentes estão em risco com o uso do método.

\section{CONCLUSÃO}

A tomografia computadorizada é um método eficaz de avaliação pós operatória da posição do parafuso iliosacral.

A maior parte dos parafusos que estavam mal posicionados em relação ao corredor, ocorreu em casos em que a redução do anel pélvico foi considerada insatisfatória, o que mostra a importância da redução do anel pélvico previamente à inserção dos implantes.

A ausência de lesões iatrogênicas em nossa série, a despeito da proporção significativa de parafusos localizados fora do corredor de segurança, nos dá a dimensão de que a fixação iliosacral é um método relativamente seguro para o tratamento das fraturas do anel pélvico.

\section{REFERÊNCIAS}

1. Routt MLC, Kregor PJ, Simonian PT, et al. Early results of percutaneous iliosacral screws placed with the patient in the supine position. J Orthop Trauma. 1995; 9: 207-214.

2. Moed BR, Hartman MJ, ahmad BK, et al. Evaluation of intrãoperative nervemonitoring during insertion of an iliosacral implant in an animal model. J Bone Joint Surg AM. 1999; 81: 1529-1537.

3. Ziran BH, Smith WR, Towers J, et al. Iliosacral screw fixation of posterior pelvic ring using local anaesthesia and computerised tomography. J Bone Joint Surg BR. 2003; 85: 411-418.

4. Carlson DA, Scheid DK, Maar DC, et al. Safe placement of S1 and S2 iliosacral screws: the "vestibule" concept. J Orthop Trauma. 2000; 14: 264-269.

5. Pennal, CF, and Sutherland, GO. Fractures of the pelvis (motion picture). Park Ridge, IL, American Academy of Ortopaedics Surgeons Film Library, 1961

6. Matta J, Dickson K, Markovich G. Surgical Treatment of pelvic nonunions and malunions. Clin Orthop. 1996; 329: 199-206.
7. Cohen MT, Guimarães JM, Filho GRM, Cohen JC, Goldsztajn, Guimarães FM. Fixação Percutânea iliosacral na lesão traumatic do anel pelvic. Rev Bras Ortop. 2005; 40: 32-41.

8. Reilly MC, Bono CM, Litkouhi B, et al. The effect of sacral fracture malreduction on the safe placement of iliosacral screws. J orthop Trauma. 2003; 17: 88-94.

9. Tile M. Acute Pelvic Fracture. I Causation and Classification. JAÃOS. 1996; 4: 143-151.

10. Barrick F, O'mara JW, Lane E. Iliosacral screw insertion using computer-assisted CT images guidance: a laboratory study. Computer Aided Surgery.1998; 3: 289-296

11. Routt MLC, Simonian PT, Agnew SG, et al. Radiographic recognition of the sacral alar slope for optimal placement of iliosacral screws: a cadaveric and clinical study. J Orthop Trauma. 1996, 10: 171-177.

12. Templeman D, Schimidt A, Freese j, et al. Proximity of iliosacral screws to neurovascular structures after internal fixation. Clin Orthop 1996; 329: 186-193. 